\title{
Fístula arteriovenosa safeno-femoral superficial como acesso à hemodiálise - descrição de técnica operatória e experiência clínica inicial
}

\author{
Superficial saphen ofemoral arteriovenous fistula as access to hemodialysis - \\ description of operative technique and initial clinical experience
}

\author{
João Antônio C orrêa ${ }^{1}$, Adilson C asemiro Pires', 0 hannes Kafejian ${ }^{3}$, Fausto Miranda Jr. ${ }^{4}$, \\ Sidnei José G alego ${ }^{3}$, Yumiko Regina Yamazaki ${ }^{5}$, Eliane Yumi Fujiii, ${ }^{6}$ Alexandre César Fioretti ${ }^{7}$
}

\section{Resumo}

O bjetivo: D escrever uma técnica de confecção de fístula arteriovenosa para acesso à hemodiálise, avaliando os aspectos técnicos de sua confecção, eficácia e complicações.

M étodo: Foram realizadas 16 fístulas arteriovenosas safenofemoral superficial em 15 pacientes, no período de agosto de 1998 a outubro de 2000. Esses procedimentos foram efetuados em pacientes sem opções de acesso em membros superiores. A técnica utilizada foi a anteriorização e superficialização da veia safena magna, anastomosando-a na artéria femoral superficial distal. As fístulas arteriovenosas safeno-femoral superficial foram avaliadas quanto à facilidade de punção, fluxo adequado, pressão venosa espontânea, adequação de diálise e complicações no intra-operatório.

Resultados: Todas as fístulas puderam ser concluídas com sucesso, sem complicações no intra-operatório. H ouve um óbito precoce, porém as demais estavam aptas às punções no $30^{\circ}$ dia pós-operatório. $Q$ uatorze fístulas foram utilizadas e, na evolução, três pacientes foram submetidos a transplante renal, quatro apresentaram trombose, dois apresentaram pseudoaneurisma de punção.

C onclusão: As fístulas arteriovenosas safeno-femoral superficial mostram-se como boa alternativa para pacientes que não possuem outras possibilidades de acesso em membros superiores, permitindo tratamento hemodialítico eficaz, com boa taxa de perviedade em mé dio prazo.

Palavras-chave: fístula arteriovenosa, hemodiálise, veia safena.

\begin{abstract}
O bjective: To describe a technique for creating an arteriovenous fistula as an access to hemodialysis, evaluating its technical aspects, efficacy and complications.

Method: From August 1998 to 0 ctober 2000, 16 superficial saphenofemoral arteriovenous fistulas were performed in 15 patients. These procedures were used in patients without access options in upper limbs. The surgical technique consisted of an anteriorization and a superficialization of the saphenous vein anastomosed to the superficial femoral artery in the distal portion. The superficial saphenofemoral arteriovenous fistulas were evaluated with regard to puncture, appropriate flow, spontaneous venous pressure, dialysis adequacy and intraoperative complications.

Results: There were no intraoperative complications and all the fistulas could be successfully concluded. There was one early death, but the other fistulas were able to the hemodialysis in the 30th postoperative day. Fourteen fistulas were used; in the evolution, three patients were submitted to renal transplant, four presented thrombosis and two presented puncture pseudoaneurysm.

Conclusion: Superficial saphenofemoral arteriovenous fistulas proved to be a good alternative for patients who do not have other possibilities of vascular access in upper limbs, thus allowing an effective hemodialysis treatment, with a good patency rate at medium term.
\end{abstract}

Key words: arteriovenous fistula, hemodialysis, saphenous vein.

1. Mestre. Médico assistente da Disciplina de Angiologia e Cirurgia Vascular, Faculdade de Medicina do ABC (FMABC), Santo André, SP.

2. Doutor. Professor titular da Disciplina de Cirurgia Cardíaca, FMABC, Santo André, SP.

3. Doutor, Professor titular da Disciplina de Angiologia e Cirurgia Vascular, FMABC, Santo André, SP

4. Doutor. Chefe da Disciplina de Angiologia e Cirurgia Vascular, Escola Paulista de Medicina (UNIFESP), São Paulo, SP

5. Mestranda de Angiologia e Cirurgia Vascular, FMABC, Santo André, SP.

6. Acadêmica do Curso de Medicina, FMABC, Santo André, SP.

7. Médico assistente da Disciplina de Angiologia e Cirurgia Vascular, FMABC, Santo André, SP.

Artigo submetido em 30.05.05, aceito em 08.11.05. 
0 método de escolha para acesso à hemodiálise éa fístula arteriovenosa (FAV) primária em membro superior, envolvendo a artéria radial e a veia cefálica na região do punho, como descrita por Brescia et al. em 1966. T al técnica eleva o fluxo sangüíneo venoso para cerca de 250 a $300 \mathrm{ml}$ por minuto, sendo essa a velocidade de fluxo ideal para se obter o clearance de uréia adequado após 4 horas de hemodiálise ${ }^{1}$.

A preferência por sua utilização deve-se ao maior tempo defuncionamento dessas comunicações arteriovenosas, à baixa taxa e ao fácil tratamento de suas complicações ${ }^{2-4}$.

A utilização prolongada desses acessos pode levar a complicações, como infecção, pseudoaneurisma de punção, pseudoaneurisma anastomótico, hipertensão venosa, isquemia distal e mesmo à sua perda ${ }^{5,6}$.

No esgotamento detodas as possibilidades de acessosem membrossuperiores, seja através deanastomoses arteriovenosas primárias, seja através de enxertos com próteseou veia autóloga, uma proposta éa confecção de FAV em membros inferiores. Para tal, são comumente utilizadas duas técnicas básicas: as que utilizam uma alça de veia safena magna ${ }^{7,8}$ ou de material protético $0^{4,9}$ e as que utilizam pontes entre a artéria ea veia femoral, empregando material sintético ${ }^{10}$.

As desvantagens dessas al ternativas são a utilização de material protético, o risco de acotovelamento das alças, a necessidade de duas anastomoses vasculares e a proximidade da região inguinal, onde o risco de infecção é maior ${ }^{2,3,9-12}$.

O s objetivos deste trabal ho são descrever uma técnica de confecção de FAV como acesso à hemodiálise, avaliar os aspectos técnicos de sua confecção e a sua eficácia durante as sessões dialíticas quanto à facilidade de punção, perviedade e complicações.

\section{M etodologia}

\section{Local de estudo}

Serviço de $\mathrm{N}$ efrologia do $\mathrm{H}$ ospital de Ensino da FU ABC, em São Bernardo do C ampo (SP) e C entro de $N$ efrologia e H ipertensão ( $C N H$ ), em Santo André (SP).

\section{Período do estudo e características da amostra}

No período de agosto de 1998 a outubro de 2000, foram realizadas 16 fístulas arteriovenosas safeno-femoral superficial (FAVSFS) em 15 pacientes em programa de hemodiálise, nos quais foram esgotados todas as possibilidades de acesso em membros superiores e que preencheram pré-requisitos do protocolo.

A amostra constituiu-se de 11 pacientes do sexo feminino (76\%) e quatro do sexo masculino (24\%). A idadevariou entre 24 e 80 anos, com média de 43,4 anos.

D os 15 pacientes, $13(86,6 \%)$ apresentavam doenças associadas, como hipertensão arterial sistêmica, cardiopatias, colagenose, dislipidemia, doença pulmonar obstrutiva crônica, diabetes melito e dependente de drogas.

0 número de fístulas confeccionadas previamente à FAVSFS variou de uma (um paciente) a oito (um paciente), sendo a mediana dequatro. Todos ospacientes apresentaram, em al guma época de sua utilização, uma ou mais complicações dessas fístulas.

\section{Variáveis estudadas}

Intensidade do fluxo: o fluxo foi avaliado pela intensidade do frêmito ao término da cirurgia.

Complicações intra-operatórias.

Complicações pós-operatórias: foram avaliadas semanalmente até $030^{\circ}$ dia pós-operatório.

Eficácia da fístula e complicações inerentes à sua utilização: iniciada após $030^{\circ}$ dia pós-operatório.

M étodo de análise: através da curva de perviedade cumulativa - life table.

\section{M étodo}

A técnica consiste em confeccionar uma FAV empregando uma única anastomose entre a veia safena magna e a porção distal da artéria femoral superficial, após sua superficialização e anteriorização.

O s pacientes devem apresentar veias safenas magnas proximais, com calibreigual ou superior a $5 \mathrm{~mm}$ de diâmetro e as artérias femorais superficiais distais com calibre entre 6 e $8 \mathrm{~mm}$.

\section{Técnica operatória}

Os pacientes foram submetidos à confecção de FAVSFS, obedecendo à seguinte padronização:

Eleição do membro mais apropriado para a confecção da FAV através de exame clínico, baseado nas condições arteriais e venosas: pulsos periféricos presentes e normaise ausência de sinais de hiperten são venosa crônica ou doença varicosa. 
Paciente posicionado em decúbito dorsal horizontal sob raquianestesia.

Incisão longitudinal de aproximadamente $10 \mathrm{~cm}$ em terço médio-distal da face interna da coxa.

D issecação por planoseisolamento da artériafemoral superficial acima do canal dos adutores, afastando medialmente o músculo sartório.

Incisão longitudinal de aproximadamente $6 \mathrm{~cm}$ na região inguinal, dissecação eligadura de veiastributárias da veia safena magna proximal.

I ncisões escalonadas para dissecação da veia safena em sua porção médio-distal de coxa.

Ligadura distal, retirada do seu leito, cateterização com sonda plástica número 6 e heparinização da veia safena com $20 \mathrm{ml}$ de solução de heparina sódica a 1\%.

C onfecção de túnel subdérmico através de dissecação romba na região ântero-lateral de coxa, de modo a anteriorizar e superficializar a veia safena em seu novo leito.

Passagem da veia safena através do túnel subdérmico (Figura 1).

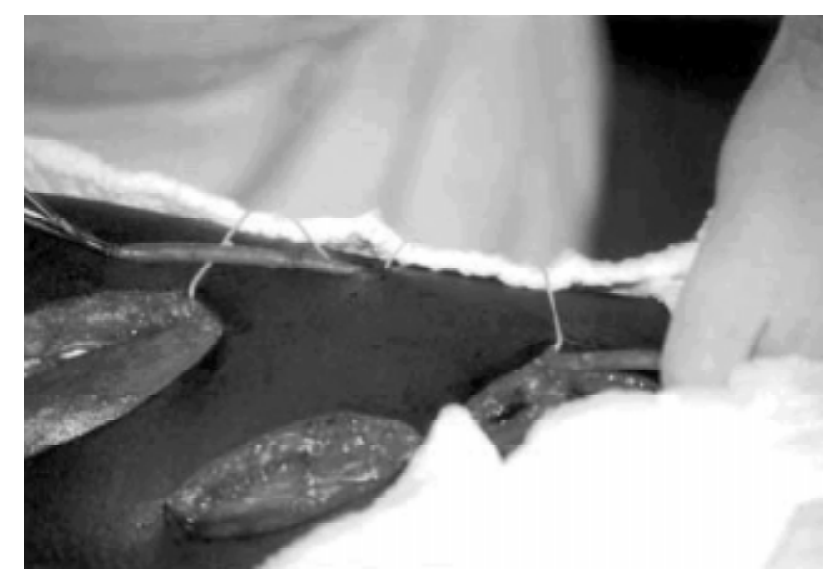

Figura 1 - Passagem da veia safena através do túnel subcutâneo

Clampeamento proximal edistal da artériafemoral earteriotomia longitudinal de aproximadamente $1 \mathrm{~cm}$.

H eparinização proximal com $10 \mathrm{ml}$ da solução padronizada, anastomose término-lateral, confecção da FAV, com sutura contínua de polipropileno 6.0 (Figura 2).

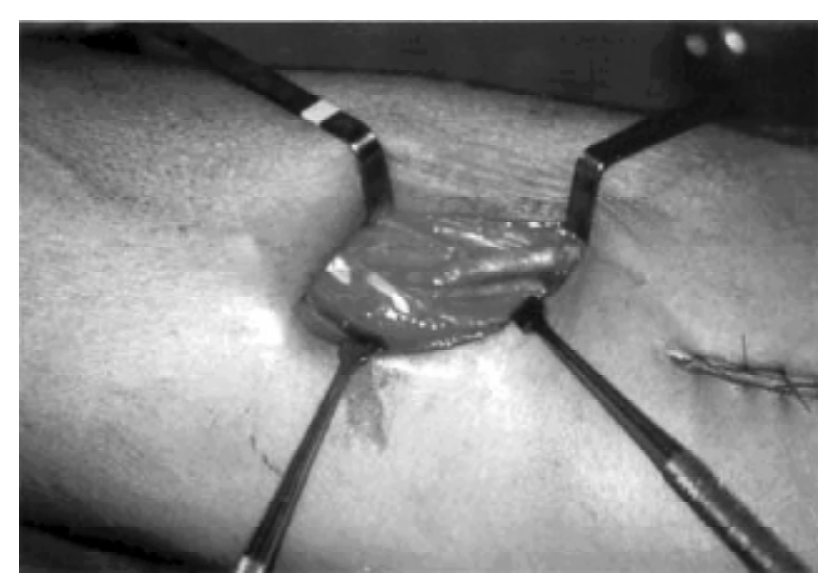

Figura 2 - Fístula arteriovenosa

D esclampeamento arterial, venoso e verificação dos frêmitos.

V erificação das condições finais, como conformação da veia e os frêmitos proximal e distal daFAV (Figura 3).

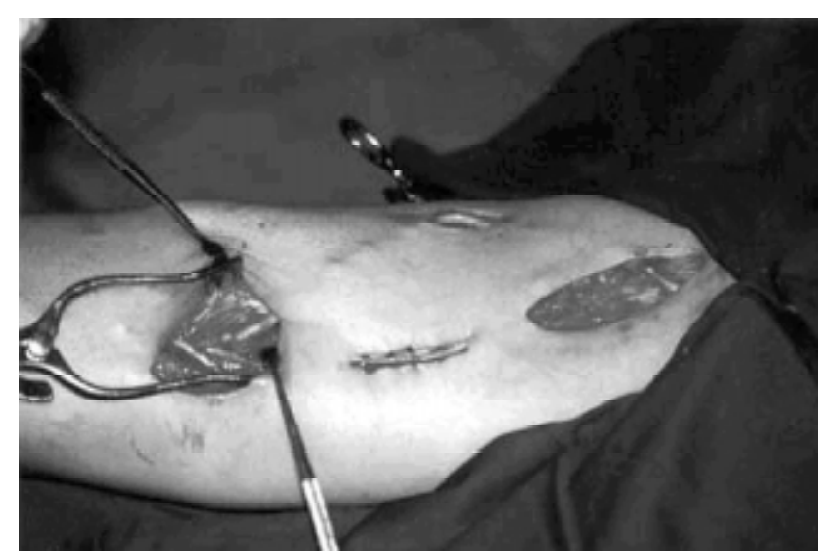

Figura 3 - Aspecto final da cirurgia

Fechamento da ferida cirúrgica por planos e realização de curativo.

\section{Controles ou seguimentos pós-operatórios}

O s pacientes receberam alta hospitalar após verificação das condições clínicas e resultado cirúrgico. 
O seguimento pós-operatório deve ser realizado ambulatorialmente na unidade de hemodiálise a cada 7 dias. Após 30 dias, a fístula poderá ser liberada para punções, após avaliação de sua perviedade.

As FAV devem ser avaliadas quanto à sua facilidade de punção, fluxo de hemodiálise, ausência de pressão venosa espontânea e adequação de diálise segundo K.T/V 13 .

D eve ser avaliada, também, a presença ou não das seguintes complicações: trombose, hematomas póspunção, isquemia distal do membro, hipertensão venosa, descompensação cardíaca, infecções, pseudoaneurisma de punção, pseudoaneurisma anastomótico e dilatação aneurismática.

Foi definida como facilidade de punção a cateterização eficaz da fístula na sua primeira tentativa. Fluxo de hemodiálise ideal, acima de $250 \mathrm{ml}$ por minuto. Ausência de pressão venosa espontânea, pressão menor que $100 \mathrm{mmH} g$ na extremidade de devolução com fluxo sangüíneo ideal. D efiniu-se como adequação de diálise o clearance de uréia (in vitro) multiplicado pelo tempo de diálise (minutos), dividido pelo volume de distribuição deuréia (peso X 0,6), considerando o valor ideal semanal de $1,2^{13}$.

O s dados foram anotados no protocolo de seguimento e compilados neste trabalho, e os resultados analisados devem ser divididos em: imediato, precocee tardio.

0 resultado imediato foi definido como a intensidade do fluxo e a presença ou não de complicações operatórias ao término da cirurgia. Resultado precoce: a evolução da fístula desde a al ta hospitalar até o início das punções após 30 dias. Resultado tardio: a evolução da fístula após o início das punções.

\section{Resultados}

\section{Resultado imediato}

Todosospacientes preenchiam as especificações do protocolo, sendo possível a realização da técnica em todos os casos.

0 membro inferior direito foi utilizado em 12 cirurgias (75\%) e o esquerdo, em quatro (25\%).

0 fluxo através da fístula, avaliado pelaintensidade do frêmito, tanto na região proximal como na distal à anastomose, na desembocadura da safena na veia femoral comum, após o desclampeamento arterial, foi considerado adequado em todos os pacientes.
$\mathrm{N}$ ão houve nenhum tipo de complicação no intraoperatório. Ao término da cirurgia, todos os pacientes apresentaram perfusão e pulsos distais do membro utilizado nas mesmas condições prévias.

Dois pacientes tiveram oclusão precoce de suas fístulas. U m, do sexo masculino, apresentou a oclusão no pós-operatório imediato em conseqüência da compressão extrínseca por hematoma no trajeto da fístula, causada por distúrbio de coagulação. N o outro, do sexo feminino, a causafoi o acotovel amento dafístula em seu novo traj eto subcutâneo, por redundância da veia safena. Ambas puderam ser corrigidas cirurgicamente, eas fístulas mantiveram-se funcionantes. Q uatorze pacientes $(87,5 \%)$ tiveram alta no primeiro dia do pósoperatório e um $(6,25 \%)$ no segundo dia.

H ouve um óbito $(6,25 \%)$ devido à coagulação intravascular disseminada no segundo dia de pós-operatório. Esse paciente, portanto, foi eliminado da série, pois não houve utilização da fístula.

\section{Resultado precoce}

N o sétimo dia depós-operatório, todosospacientes apresentavam a cicatriz cirúrgica sem sinais de infecção e com suas fístulas funcionantes, sem complicações clínicas, arteriais e/ou venosas no membro utilizado.

No $30^{\circ}$ dia de pós-operatório, todas as fístulas apresentavam-se funcionantes e aptas às punções. Em um paciente, optou-sepor manter a utilização defístula de PTFE em membro superior, que anteriormente se apresentava com infecção e, nessa ocasião, mostrava-se total mente curada. Foi, portanto, excluído da avaliação da eficácia dessa técnica.

As 14 fístulasutilizadas mostraram-seeficazes. N ão apresentaram dificuldadedepunção, apresentaram bom fluxo de hemodiálise (300 ml/min), não apresentaram pressão venosa espontânea e a adequação de diálise segundo K.T/V dentro do valor ideal ${ }^{3}$.

\section{Resultado tardio}

T rês pacientes foram submetidos a transplante re nal, com rim de cadáver (23\%), sendo um do sexo masculino e dois do feminino. O s transplantes ocorreram no terceiro, quinto esétimo mês, respectivamente, após confecção das fístulas.

N o seguimento deaté 26 meses, das 11 fístulas que foram utilizadas, nos 10 pacientes que mantinham um programa de hemodiálise, foram observados quatro 
tipos de complicações: trombose, pseudoaneurisma de punção, hematoma de punção e estenose.

A trombose ocorreu em quatro fístulas $(36,4 \%)$, sendo duas em pacientes do sexo feminino, no segundo e sexto mês de suas confecções, e duas em pacientes do sexo masculino, no oitavo e $16^{\circ}$ mês de suas confecções, respectivamente. 0 pseudoaneurisma de punção acometeu duas fístulas (18,2\%), ambos em pacientes do sexo feminino, 4 meses apóssuas confecções, em ambos os casos.

0 hematoma de punção e a estenose ocorreram em uma fístula $(9,1 \%)$, no terceiro e sétimo mês de evolução, respectivamente, em pacientes do sexo feminino.

O s pacientes que apresentaram como complicação o pseudoaneurisma de punção puderam ser tratados cirurgicamente, por meio deressecção einterposição de um novo segmento de safena através de anastomoses término-terminal com fios de polipropileno 6.0 (enxerto safeno-safeno com safena), mantendo a perviedade secundária das fístulas e, conseqüentemente, a sua utilização.

N o caso da estenose, a perviedade secundária também pode ser mantida através da angioplastia transluminal percutânea, realizada após o diagnóstico de estenose ter sido confirmado pela fistulografia por subtração digital.

O hematoma de punção foi tratado clinicamente, e sua absorção ocorreu espontaneamente, não comprometendo a perviedade da fístula. Inicialmente, após o tratamento das complicações, oito fístulas $(72,7 \%)$ puderam ser utilizadas cronicamente. $\mathrm{N}$ o entanto, esse número caiu para sete $(63,3 \%)$ após a oclusão de uma das fístulas em que foi realizada a correção do pseudoaneurisma.

$\mathrm{N}$ os 26 meses de acompanhamento, houve apenas um óbito, por hemorragia digestiva, em uma paciente do sexo feminino, 7 meses após a confecção de fístula, que se mantinha funcionante até essa ocasião.

Até o momento, seis fístulas continuam sendo utilizadas, cinco em pacientes do sexo feminino eum do sexo masculino. Todas se mostram eficazes e possibilitam diálises adequadas.

Baseando-se na T abela 1, realizou-se análise da perviedade cumulativa (life-table), com um resultado de $64 \%$ de perviedade provável ao término desse período, com um desvio padrão acima de $10 \%$ após 14 meses de evolução, conformeilustração gráfica na Figura 4.
Tabela 1 - D istribuição dasfístulasutilizadas segundo sexo, idade e evolução com relação ao tempo de confecção

\begin{tabular}{lcccc}
\hline Caso & Sexo & $\begin{array}{c}\text { Idade } \\
\text { (anos) }\end{array}$ & Evolução & $\begin{array}{c}\text { Tempo de } \\
\text { confecção } \\
\text { (meses) }\end{array}$ \\
\hline 1 & $\mathrm{~F}$ & 36 & Pérvia & 26 \\
2 & $\mathrm{~F}$ & 57 & Pérvia & 25 \\
3 & $\mathrm{M}$ & 45 & Trombose & 16 \\
4 & $\mathrm{~F}$ & 80 & Pérvia & 12 \\
5 & $\mathrm{M}$ & 39 & Trombose & 8 \\
6 & $\mathrm{~F}$ & 28 & Transplante & 7 \\
7 & $\mathrm{~F}$ & 38 & Ó bito & 7 \\
8 & $\mathrm{~F}$ & 51 & Trombose & 6 \\
9 & $\mathrm{~F}$ & 38 & Transplante & 5 \\
10 & $\mathrm{~F}$ & 47 & Pérvia & 5 \\
11 & $\mathrm{M}$ & 26 & Transplante & 3 \\
12 & $\mathrm{M}$ & 43 & Pérvia & 3 \\
13 & $\mathrm{~F}$ & 24 & Trombose & 2 \\
14 & $\mathrm{~F}$ & 24 & Pérvia & 2 \\
\hline
\end{tabular}

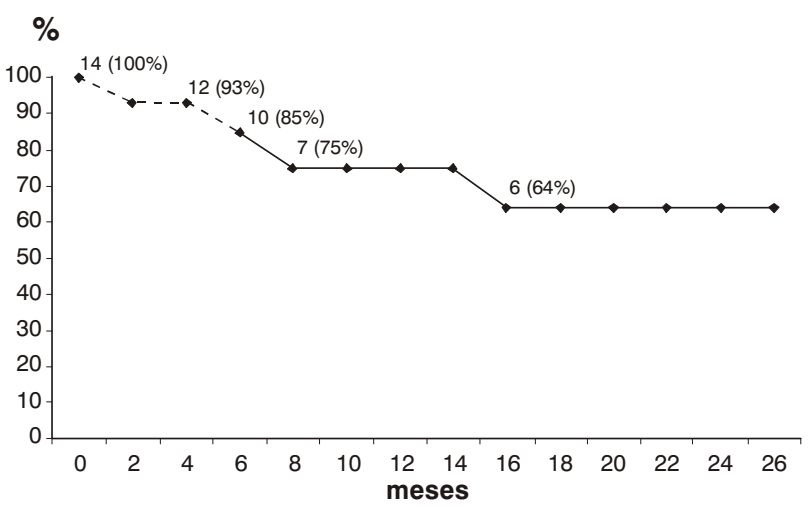

Figura 4 - Curva de perviedadecumulativa (life table) em 26 meses

\section{D iscussão}

A hemodiálise é fundamental para a sobrevivência do portador de insuficiência renal crônica, pois a única possibilidadede cura éo transplante. N o entanto, a taxa de pacientes que conseguem esse tipo de tratamento, seja de doadores vivos ou de cadáveres, é muito baixa, necessitando, portanto, permanecer em programa dialítico, na maioria das vezes por muitos anos.

A falência dos acessos à hemodiáliseéa maior causa de morbidade para os pacientes em programa dialítico. 
Vários relatos indicam que a alta porcentagem de hospitalização do paciente renal crônico éem função de complicações do acesso vascular, eque o intervalo entre as internações desses pacientes diminuem à medida que se esgotam as possibilidades desses acessos, elevando significativamente o custo final, tanto clínico quanto financeiro, que chega a ser de um bilhão de dólares anuais nas estatísticas norte-americanas ${ }^{14}$.

I sso nos motivou a buscar uma nova alternativa de acesso à hemodiálise, em membros inferiores, para os casos em que todas as possibilidades em membros superiores estivessem esgotadas. Procuramos uma técnica de menor custo, eficaz, que tivesse longa duração e com baixa taxa de complicações.

$N$ este estudo, foram incluídos pacientes nos quais houveesgotamento detodas aspossibilidades deacessos em membros superiores. Com a utilização da veia safena magna autóloga, evitou-se o uso de material sintético, como o politetrafluoretileno (PTFE), que sabidamenteaumentaaincidência deinfecção eimplica em um custo muito maior $4,9,10,12$.

0 utro grande benefício da utilização da veia safena na FAV em coxa foi a necessidade de apenas uma anastomose, o que diminui as complicações inerentes a ela.

A escolha do sítio de anastomose na artéria femoral superficial distal, acima dos canais dos adutores, teve a intenção de evitar 0 acotovelamento da safena durante a sua acomodação no túnel subcutâneo, além demantêla distante da região inguinal, onde o risco de infecção é maior 2-4,9,11,12. 0 utra vantagem é que essa artéria é defácil acesso efacilita muito a confecção da anastomose. N essa técnica, a anastomose fica protegida pelo músculo sartório, evitando punções próximas a ela e também diminuindo os riscos de pseudoaneurismas anastomóticos.

$\mathrm{N}$ a técnica com conformação em ponte, com a veia safena anteriorizada esuperficializada, permite, além de maior facilidade para punção, um extenso segmento venoso, o que evita as punções repetidas em um mesmo local, diminuindo assim o risco de pseudoaneurisma, trombose e estenoses precoces ${ }^{9,15}$. Além disso, possibilita para o paciente uma posição confortável e segura durante a hemodiálise.

A complicação mais grave emais comum em nosso estudo foi a trombose tardia da fístula, ocorrida em quatro pacientes $(28,6 \%)$. Em dois deles foi provavelmente em conseqüência das punções repetidas em um mesmo local, provocando a estenose, hipofluxo e posterior trombose ${ }^{15}$.

0 diagnóstico de punções repetidas pode ser realizado através do exame clínico, no qual os pacientes apresentavam uma área de hipopigmentação de pele e nódulo endurecido de segmento venoso nessa área correspondente. Os outros dois casos de trombose ocorreram em pacientes do sexo feminino, sendo que, em um deles, aconteceu após trauma no local da fístula e, no outro caso, ocorreu 2 meses após a sua confecção. A causa mais provável foi a hipotensão.

A outra complicação encontrada, o pseudoaneurisma de punção, ocorreu em dois pacientes $(14,3 \%)$, podendo ser diagnosticado clinicamente e confirmado através de fistulografias. Essa complicação deu-se provavelmente em razão da compressão inadequada do local de punção após as sessões de diálise, associada a punções repetidas em um mesmo local, levando à fraqueza da parede da fístula. N o entanto, foi possível corrigir esse tipo de complicação através de sua ressecção e interposição de um novo segmento de safena, mantendo a perviedade secundária da fístula.

0 caso de hematoma pós-punção também ocorreu devido à compressão e cuidado inadequado após sessão dialítica. Essa fístula pode ser preservada por meio de tratamento clínico e absorção espontânea do hematoma. No entanto, o risco de ocorrer uma infecção é sempre grande nesses casos.

0 caso de estenose foi detectado pela queda do fluxo durante as sessões de diálise. Esse fato pode ser confirmado através de fistulografia por subtração digital. Essa complicação pode ser corrigida pela terapêutica endovascular, por dilatação transluminal percutânea, manten do a perviedadeda fístula, ea sua utilização pode ser retomada no dia seguintea esse procedimento.

$T$ ais fatos ocorreram devido à pouca intimidade na manipulação desse novo procedimento, tanto pela enfermagem quanto pelo paciente. $\mathrm{N}$ essa fístula, por utilizar uma artéria de alto fluxo, os cuidados de punção, variação e compressão desses locais deverão ser maiores que o habitual nas fístulas de membros superiores ${ }^{16}$.

I sso nos alerta para maior cuidado, melhor orientação ao corpo de enfermagem da unidade de diálise, ao paciente e aos familiares quanto a esses problemas.

Com relação às tromboses, tais cuidados também terão que ser observados, pois essas raramente poderão ser revertidas. N o entanto, poderão ser evitadas, desde 
que as estenoses sejam precocemente diagnosticadas e corrigidas.

U ma vez ocorrida a trombose, poderá ser indicada a confecção denovaFAV SFS no membro contral ateral, desde que a veia safena o permita.

O utras complicações, como isquemia distal, hipertensão venosa, descompensação cardíaca, pseudoaneurismas anastomóticos, dilatações aneurismáticas e outros tipos de infecções, não foram observadas até a presente data.

N o momento, seis fístulas $(42,8 \%)$ estão sendo utilizadas como acesso à hemodiálise, mostrando-se eficazes quanto ao fluxo ideal ( $300 \mathrm{ml} / \mathrm{min}$ ), com baixo índice de pressão venosa espontânea e com adequação de diálise segundo $\mathrm{KT} / \mathrm{V}$ ideal ${ }^{13}$.

$\mathrm{Na}$ análise dos resultados imediatos e precoces, pudemos observar uma taxa de sucesso de $100 \%$ em ambas as situações, o que nos animou a continuar investindo nessa nova técnica.

$\mathrm{N} \mathrm{a}$ análise do resultado tardio, observamos que $42,8 \%$ das fístulas mantêm-se funcionantes e que, adicionado aos pacientes transplantadoseao óbito, que apresentava sua fístula pérvia, podemos considerar uma alta taxa de sucesso desse procedimento.

Através da análise da curva de perviedade cumulativa (life-table) em 26 meses, pudemos observar uma taxa de $64 \%$ de probabilidade de perviedade nesse período ${ }^{17-19}$. Essa taxa provavelmente seria maior, caso não tivessem sido excluídos os pacientes transplantados. 0 desvio padrão elevado (acima de 10\%) após 14 meses de evolução, observado na análise da curva, foi influenciado pelo pequeno tamanho da amostra17-19.

A limitação a essa técnica está nos casos em que o pacienteé safenectomizado ou apresenta a safena interna inadequada para essa utilização e nos pacientes portadores de arteriopatia oclusiva no território fêmoro-poplíteo.

0 utra crítica que pode ser feita a essa técnica é que a safena, por sua camada muscular desenvolvida, impede o desenvolvimento da fístula, como ocorre na veia cefálica nas FAV de antebraço. I sso, apesar de evitar a dilatação aneurismática, aumentará o risco de hiperplasia miointimal após punções repetidas da FAV ${ }^{15}$.

$\mathrm{N}$ o entanto, por ser material autólogo, de baixo custo, demaior resistênciaa infecçõesemaior facilidade etratamento dasmesmas, as vantagenssobrepujam suas deficiências.
A experiência está em sua fase inicial, e a casuística é pequena, com pouco tempo de acompanhamento, 0 que impossibilita uma análise estatística mais rigorosa quanto à sua evolução a médio e longo prazo.

\section{Conclusões}

A FAVSFS apresenta-se como alternativa viável em pacientes que não possuem mais opções de acesso vascular em membro superior, com boa eficácia, baixa morbidade, complicações passíveis de correção e boa taxa de perviedade em médio prazo.

\section{R eferências}

1. Brescia M, Cimino JE, Appel K, Hurwish BJ. Chronic hemodialysis using venipuncture and surgically created arteriovenous fistula. N Engl J M ed. 1966;275:1089-92.

2. Bhandari S, W ilkinson A, Sellars L. Saphenous vein forearm grafts and gortex thigh grafts as alternative forms of vascular access. Clin N ephrol. 1995;44:325-8.

3. Santiago-D elpin EA, Buselmeier TJ, Simmons RL, N ajarian JS, Kjellstrand CM . A modified saphenousvein loop-fistulain the thigh as vascular access for hemodialysis in patients with access problems. Ann Royal Coll Surg Engl. 1987;70:244-5.

4. Slater ND, Raftery AT. An evaluation of expanded polytetrafluorethylene (PTFE) loop grafts in the thigh as vascular access for hemodialysis in patients with access problems. Ann Royal Coll Surg Engl. 1987;70:244-5.

5. Galego S]. Estudo comparativo de fluxo de fístulas arteriovenosas femorais termino-lateral e latero-lateral modificada em cães [dissertação]. São Paulo: Escola Paulista de M edicina, U niversidade Federal de São Paulo, 1988.

6. Holman E. Clinical and experimental observations and arteriovenous fistula. Am Surg. 1940;112:840-75.

7. K innaert $P, V$ ereestraeten $P, T$ oussaint $C$, van $G$ ertru $Y$ den J. Saphenous vein loop fistula in the thigh for maintenance hemodialysis. W orld J Surg. 1979:3:95-8.

8. M ay J, Tiller D, Johnson J, Stewart J, Ross Sheil AG. Saphenous vein arteriovenous fistula in regular dialysis treatment. N Engl J Med. 1990;280:770.

9. Khadra $\mathrm{MH}$, D wyer AJ, Thompson JF. Advantages of polytetrafluorethylene arteriovenous loops in the thigh for hemodialysis access. Am J Surg. 1997;173:280-3.

10. W ilson SE, Stabile BE, W illiams RA, O wens M L. Estudo atual das técnicas de acesso vascular. In: Connoly JE. Clínica C irúrgicadaA méricado N orte. Rio deJ aneiro: Interamericana; 1982. p. 595-617.

11. Lynggaard F, N ordiling J, H ansen RI. Clinical Experience with the saphena loop arteriovenous fistula on the thigh. Int U rol N ephrol. 1981;13:287-90.

12. W ilson SE, Hillman M, O wens M L. Hemodialysis hemodynamic effects of bovinefemorosaphenousfistula. Dial Transplant. 1977;6:84. 
13. Barth RH . D irect cal culation of KT/V . An amplified approach to monitoring of hemodialysis. N ephron. 1988;50:191-5.

14. M iller PE, Tolwani A, Luscy CP, D eierhol M H, Bailey R, Redden $\mathrm{DT}$, Allon M . Predictors of adequacy of arteriovenous fistulasin hemodialysispatients. Kid Internat. 1999;56;275-80.

15. N eyra N R, I kizler TA, M ay RE, H immelfarb J, Schulman G, Shyr $Y, H$ akim RM . Change in access blood flow overtime predicts access thromboses. Kid Internat. 1998;54:1714-19.

16. N KF DOQI - Clinical practice guidelines for vascular access. N ew York (USA); 1997. Am J Kid D is. 1997;30(4 Suppl): 150-89.

17. Peto $R$, Pike $M C$, Armitage $P$, et al. D esign and analysis of randomizes trials requiring prolonged observations of each patient. Introduction and design. Br J Cancer. 1976;34: 585-612.
18. Peto R, Pike M C, Armitage $P$, et al. D esign and analysis of randomizes trials requiring prolonged observations of each patient. II. Analysisand examples. BrJ C ancer. 1977;35:1-39.

19. Rutherfor RB, Fanigan DP, Gupta SK, et al. Suggested standards for reports dealing with lower extremity ischemia. J V asc Surg. 1986;4:80-9.

Correspondência:

João Antônio C orrêa

Rua $\mathrm{M}$ anaus, 151, Vila Alzira

CEP 09195-000 - Santo André, SP

E-mail: correajoao@terra.com.br

\section{Atualização de endereços}

Prezado associado,

Sevocêmudou deendereço enão comunicou a SBACV , seu exemplar está sendo enviado ao endereço antigo. É fundamental que envie seu novo endereço à Secretaria da SBACV para garantir o recebimento do J V asc Br.

SBACV - Sociedade Brasileira de Angiologia e C irurgia Vascular

Av. T ancredo N eves, 1632 salas 501/502

Condomínio Salvador T rade C enter

CEP 41820-020 - Salvador, BA

Fone: (71) 3271.5368

E-mail: secretaria@sbacv-nac.org.br 